\title{
Multi-scale Studies and the Ecological Neighborhood
}

\author{
Jeffrey D. Holland ${ }^{1} \cdot$ Shulin Yang $^{2}$
}

Published online: 10 September 2016

(C) Springer International Publishing AG 2016

\begin{abstract}
Multi-scale landscape studies are becoming a wellused way to examine the influence of environmental, landscape, or habitat factors on the abundance or occurrence of species. Multi-scale studies are especially useful when the ecological neighborhood of the organism-landscape interaction is unknown. We review the development of multi-scale approaches and further clarify the associated terminology applied to different aspects of spatial scale. In particular, we argue that ecological neighborhood and analytical focus are not equivalent, although analytical focus may be used to infer ecological neighborhood. We suggest several potential improvements to multi-scale landscape studies that remain to be explored. Results from multi-scale studies could be used to guide ecologically sustainable landscape planning by identifying local management practices that are suited to the landscape context.
\end{abstract}

Keywords Characteristic scale · Focus · Grain · Multi-scale · Scale of response $\cdot$ Spatial scale

This article is part of the Topical Collection on Scale-Measurement, Influence, and Integration

Jeffrey D. Holland

jdhollan@purdue.edu

1 Department of Entomology, Purdue University, 901 W. State St., West Lafayette, IN 47907, USA

2 School of Life Sciences, Guizhou Normal University, 180 N. Baoshan Rd., Guiyang, Guizhou 550001, People's Republic of China

\section{Introduction}

The use of multiple-scale studies and the interest in determining the most appropriate spatial scales for investigating species-environment relationships has increased in recent decades $[1,2]$. The past forty years have seen a great increase in explicit consideration of not only the spatial aspects of scale [3-6] but also the temporal scales associated with ecological processes [7, 8]. Examination of the spatial scaling of different ecological processes continues to reveal the hierarchy of relationships that determine species' geographic range and abundance. Many of the most important current ecological questions [9] are directly related to understanding the scale of ecological processes (e.g., "How local is adaptation?").

There continues to be interest in finding the spatial scales at which phenomena occur in order to conduct ecological research at appropriate scales for the objects under study [7, 10]. For example, when studying the influence of the patterning of land use on the abundance of a species, what sized areas should be used? Using an area too large or too small to be relevant to the processes that determine abundance of the organism could lead to an incorrect failure to reject the null hypothesis of no effect. The techniques available for species-environment investigations that seek to find relevant spatial scales also continue to grow apace [e.g., 11]. One type of investigation of spatial scaling involves the determination of the scale at which species respond to aspects of their environment. The determination of spatial scales that are relevant to ecological interactions may be accomplished by using replicated focal patches wherein the species data is determined and environmental or landscape data is gathered from larger areas of varying radii around the focal patches. This type of multiscale approach is a natural progression from studies that use replicated landscapes around focal study patches [12] and from multi-scale 'zooming' which examines dependent and 
independent variables in the same, but varying sized, areas [13]. This multiple scale adaptation of the focal patch approach [12] may have been inspired by the ecological neighborhood concept (sensu [7]).

Our objectives for this review are to: 1) briefly outline the history of multiple scale studies to highlight the links between recent developments and the ecological neighborhood, 2) use this history as context for suggesting nomenclature for various aspects of spatial scale to avoid confusion, 3 ) suggest that the scale of an ecological interaction and the scale best used to examine such an interaction is different both in concept and in magnitude by examining mechanistic determinants of analytical foci, and 4) suggest several fruitful avenues for improving multiple scale ecological studies.

In ecological studies the term scale is used to invoke a collection of related measures (Table 1) and many researchers have called for a more careful use of the term, with most agreeing that the specific aspect of scale should instead be used rather than the ambiguous term scale [16]. The most commonly invoked aspects of scale are referred to as grain $[14,15]$ and extent $[14,16]$. We will promote in this paper the seldom-used term (but see [17]) focus. Focus is the the area to which grains are aggregated to form a study replicate [18]. Part of the confusion in ecological scaling arises because the terms focus and grain are both used to refer to the size of the study replicates. These two terms should be used to describe different entities: focus for the size of study replicates and grain for the smallest distinguishable analytical unit or the smallest area whose characteristics evoke an ecological response. It is important to distinguish among scale terminology applied to the phenomenon under study, the units of information used in analyses (e.g., in GIS layers), and the ecological interactions under study $[16,19]$. This is especially important with grain and focus because the response grain may in some cases be more closely analogous to analytical focus than to the analytical grain. We may therefore, define the focus as a measure used in analyses to understand ecological interactions between organisms and their environment and as the size of the aggregation of information collected within grains into study replicates. A study replicate here would consist of a habitat patch or defined area of habitat for example, and the associated quantities of species abundance and any environmental measures of interest. The dimension of the units within which data are taken is the study grain (e.g., quadrats or map pixels) and the dimension to which the data are aggregated for that study replicate (e.g., the habitat patch) is the focus (e.g., proportion of $30 \times 30 \mathrm{~m}$ pixels of forest cover within $1 \mathrm{~km}$ ). Other terms that have been used in the literature to indicate what focus has been defined as [18] include: local extent [15], characteristic scale [20], environmental context [21], scale of effect [10], and spatial extent [22].

We suggest the adoption of new terms to refer to aspects of scale in ecological studies to clarify usage. By including both the type of measure (e.g., grain or focus) and the realm of its usage (i.e., analytical unit or scale of ecological interaction) we can clarify not only the meaning of focus (analytical focus; [18]) but also resolve the uses of the term grain: response grain for the on-the-ground area or resolution at which the study organism differentiates habitat for example [7], analytical grain for map pixels, and analytical focus for the size of study replicates (Fig. 1). Ideally then, investigations using a given analytical focus will uncover the important ecological neighborhood at which an ecological relationship 'operates.' Ecological neighborhood and the analytical focus are distinct quantities because they need not have the same magnitude although the latter may give an indication of the former. The use of these binomial terms is less convenient than focus, grain, or scale, but they are more clear in the measure being referred to. Most importantly, these terms make clear the separation of the scale of ecological interactions and the scale at which the patterns of abundance resulting from these interactions should be studied.

Early studies that made an explicit link between the area of the study replicate and the area relevant to ecological interactions explicitly considered spatial scale by examining ecological relationships at several discrete temporal and spatial magnitudes. A study of white-footed mice and meadow voles in different habitats found that microhabitat factors did not predict abundance when other selection pressures from larger foci were included [23]. A study on two simulated species using a spatially explicit cellular automaton model at a small scale and two larger scales defined by the ecological processes of interspecific interaction and of dispersal found interactions between the scale of interspecific interaction (response grain) and scale of dispersal (ecological neighborhood) [24]. The
Table 1 Spatial scale-related terminology

\begin{tabular}{lll}
\hline Term & Definition & Reference \\
\hline Grain & The size of the smallest unit in the study. & {$[14]$} \\
& $\begin{array}{l}\text { The lower boundary of resolution at which species-environment relationships can be } \\
\text { resolved. }\end{array}$ & {$[15]$} \\
Extent & The area of the full region under study. & {$[16]$} \\
& May delimit the entire time period of the study. & {$[15]$} \\
Range & The distance between study units, & \\
& Termed 'lag' in geosatistical analyses. & \\
\hline
\end{tabular}




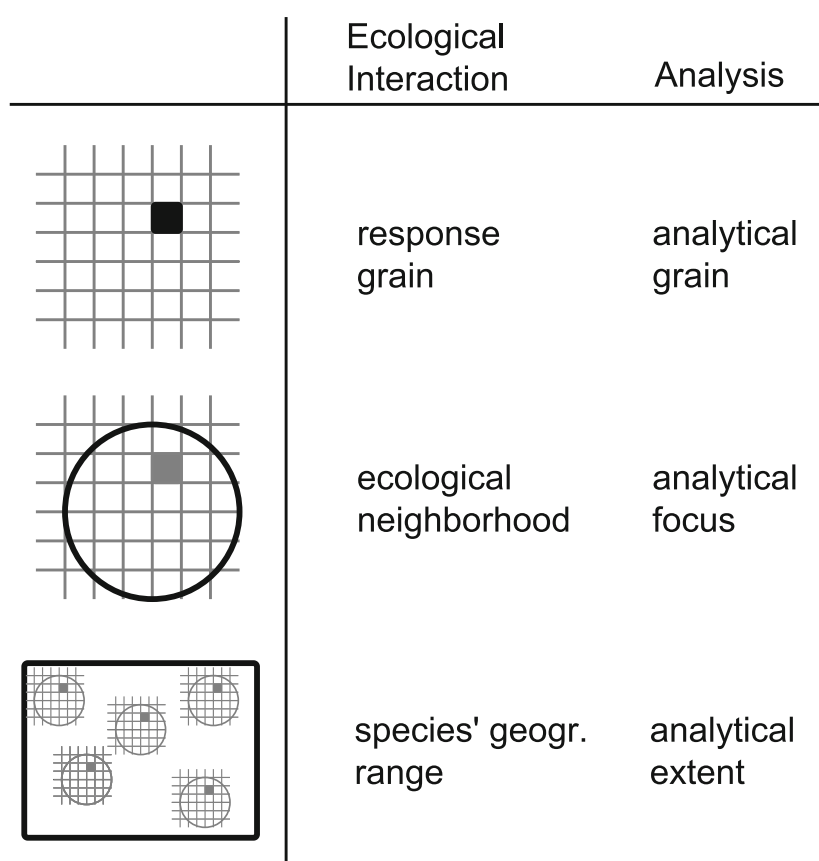

Fig. 1 Existing and suggested new terminology for different quantities in ecological studies. The features in black represent the quantity being invoked by the term while features in gray provide context. Response grain and ecological neighborhood are investigated with analytical grains and analytical foci, respectively, although the magnitude of the ecological and corresponding analytical quantity may be different. Response grain, resolution at which an organism distinguishes heterogeneity. Analytical grain, smallest unit of area in the analysis. Ecological neighborhood, area within which an interaction occurs or distance at which an organism is influenced. Analytical focus, area to which analytical grains are aggregated to form replicates in analysis. Species' geographic range, entire area occupied by individuals within a species. Analytical extent, total area from which data are taken in a study

recognition that different phenomena alter communities and abundance of single species has also been recognized in aquatic habitats. Aquatic macroinvertebrates were studied to examine the influences on these communities of factors prevalent within five differently sized areas approximating different analytical foci from the local habitat to the ecoregion [25]. Different factors influenced invertebrate abundance at different analytical foci.

Studies of a single species have been carried out at different 'scales' because different processes occur within different envelopes of time and space. For example, deer mice forage and disperse within differently sized ecological neighborhoods [26]. The distances within which deer mice forage and dispersal were found to be approximately $60 \mathrm{~m}$ and $140 \mathrm{~m}$, respectively. Short dispersal on the order of $140 \mathrm{~m}$ is unlikely to allow for selecting among many potential home sites and suggests that the role of habitat selection on local population dynamics may be overestimated [26]. This result highlights the importance of understanding how different factors influence individuals at different spatial foci and how such interactions influence the population and thus observed density.
Clearly, the interest in using study replicates of different sizes, and the recognition that different processes occur within areas of different sizes, is common across many taxa and types of habitat. The ecological neighborhood of similar processes as inferred from analytical foci can vary widely amongst different taxa. For example, the abundance of longhorned beetles was found to respond to proportion forest cover at analytical foci from $20 \mathrm{~m}$ to $2000 \mathrm{~m}$ depending on the species [20].

\section{The Ecological Neighborhood and Focal Patch Studies}

The ecological neighborhood was developed as a way to delineate the area relevant to an ecological interaction [7]. This construct built upon the work of several previous researchers in this area (e.g., [27-32]). Much of the early work that led to the formation of the ecological neighborhood as an ecological construct came from a mechanistic view of the impacts of adjacent plants on each other. There has long been discussion of the impacts of plant density on plant growth, but plants are influenced by their immediate neighbors through limited water, light, and nutrients rather than through density per se [33]. The ecological neighborhood of an organism was defined as the region within which the organism is active or influences or is influenced by a given ecological process during a time period associated with the process [7]. It was a term for describing the areal extent of an environmental interaction.

The focal patch approach to organism-environment studies compares data on the organism in replicated focal patches and the larger environment or landscape within a larger analytical focus (Fig. 2a) [12]. Varying some aspect of spatial scale, usually analytical grain or analytical focus, is one method of examining relationships between organisms and landscapes or environmental gradients to uncover the ecological neighborhood. The first way that such species-environment relationships were examined was to use an appropriate statistical model of the form Species $=\mathrm{f}$ (Environment) with measures of interest taken on species (e.g., occurrence, density, nest success) and the environment (e.g., habitat amount, food abundance) within the same sized replicate or window. The window size may then be altered or 'zoomed' (sensu [13]) and some measure of the model fit and the nature of the relationship can be examined across window sizes (Fig. 2b). The relevant measure of the nature of the relationship within each individual window size may be the slope for example. For this type of study, the size of the window is the same for the study organism and the environment or landscape. Ideally, the zooming approach allows researchers to find the ecological neighborhood at which an ecological relationship 'operates' and to examine the change in the relationship or the degree to which the signal reaching the researcher from the ecological relationship is attenuated at increasingly remote analytical foci 


\begin{tabular}{lll}
$\begin{array}{lll}\text { Study } & \text { Foci for Organism, } \\
\text { Type } & \text { Dependent Variable } & \text { Foci for Environment, } \\
\text { Independent Variable }\end{array}$ \\
\hline
\end{tabular}
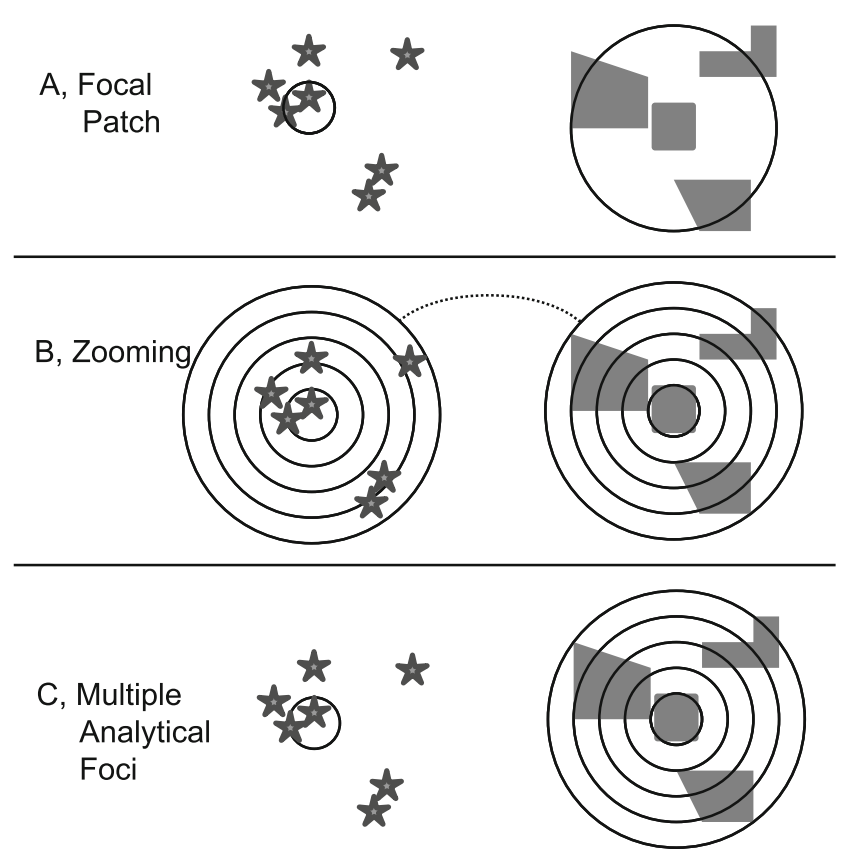

Fig. 2 Multiple scale studies for examining landscape-organism relationships. Different analytical foci are represented as differently sized circles within which the species or landscape is viewed. The data on the study organism and the data on the environment or landscape are collected within circles centered on the same point (i.e., in the same landscape) but these are shown in separate columns here for clarity. (a) Multi-scale focal patch study in which the data on the study organism come from replicated focal habitat patches (one shown here) and the landscape or environmental predictor data come from a larger analytical focus centered on the focal patch. (b) Zooming study design in which the landscape and species data are viewed within several different analytical foci, however the species data are related to landscape data within the same analytical focus as indicated by the dotted line. (c) Multiple analytical foci focal patch study approach in which species data come from replicated focal patches or areas of the same size and the landscape data come from several different analytical foci centered on the focal habitat patches

(e.g., [18]). The zooming approach to spatial scaling was used in a study examining correlations between seabirds and their prey [34]. It was found that the relationship became apparent at a particular window size and was not apparent at larger or smaller window sizes (Fig. 2b). The window size, or analytical focus, at which the seabird abundance-prey fish abundance relationship was strongest was assumed to represent the response grain, the area relevant to the decision-making process of the seabirds. This highlights the fact that whether the best analytical foci reflects the response grain or the ecological neighborhood must be decided based upon the ecological process under study.

The interest in the influence of pattern on ecological processes was brought to the forefront by landscape ecologists, who have increased usage of the habitat patch and of the landscape as study units $[35,36]$. Landscape ecological research has been aimed at understanding the impacts of landscape pattern on the abundance or occurrence of a species or the diversity within a taxon. For example, a study on birds found that the responses of bird species to vegetation characteristics of forest patches and to structural and vegetation characteristics of the surrounding forested landscapes differed among species [37]. The vegetation characteristics within the larger or smaller analytical foci were more important depending on the species of bird. Analytical foci have been used to examine much larger areas as well [e.g., 38].

The focal patch approach has been extended to use various analytical foci $[12,36]$. This extension of the focal patch approach differs from the earlier zooming approach in that the analytical foci of the dependent and independent variables are not the same. The analytical focus of the dependent variable is fixed at the habitat patch, for example, while the analytical focus of the independent variable is measured within a different, usually larger, area. The focal patch approach was proposed as a way to use landscapes as replicates because experimental manipulation of such units are unlikely due to logistical constraints. For each study unit (i.e., replicate) a dependent variable such as species' abundance is measured within the focal patch or within a fixed area. The independent variable is an environmental or landscape measure taken within an area of different size than the dependent variable. Each study replicate thus consists of patch and landscape measures at a location and each replicate represents a different landscape. Focal patch studies are called multi-scale because they investigate the dependent variable and the landscape independent variable at different 'scales' (analytical foci; [12]). In comparing this type of study with the zooming of Schneider [13] (Fig. 2b), the importance of having another aspect of scale besides grain and extent becomes clear. When increasing the size of the window of observation, it may seem convenient to simply consider this an increase in the analytical grain of the study. However, the original data may still exist at the smaller resolution, regardless of how they are aggregated as the window size increases (e.g., by taking a mean). The original analytical grain of the data conveys information on the precision and detectable heterogeneity of the data that the analytical focus and extent (and the ecological neighborhood) cannot.

An alteration to the focal patch approach allows for the detection of the analytical focus that maximizes the degree to which the actual strength of the organism-landscape relationship is detected by a study. The analytical focus of the response is held constant (e.g., abundance of a species in similarly-sized focal ponds), while the analytical focus of the predictor landscape variable is altered through some range of sizes (Fig. 2c; [12]). The idea that ecological neighborhoods have different sizes for different activities [7] leads logically to such an approach, as one analytical focus will not be suited for all ecological interactions. Landscape features necessary for daily foraging will be important within a smaller area than 
those features that allow for population persistence. This type of study allows the relevant analytical focus to be identified [20] so that the strength of the species-environment relationships are reflected accurately by the results at the appropriate analytical focus. Using a smaller or larger analytical focus would lead to attenuation of the measured relationship and a less accurate measure of the importance of the environment in determining the species' response.

The progression of studies aimed at uncovering the area representing species' response grains and ecological neighborhoods has led to multiple scale study approaches. 'Multiscale' is now usually taken to mean that the analytical focus is varied through some range of sizes, despite its former usage [12] to mean that the species and environment are measured within different analytical foci whether or not these are varied.

\section{Multi-scale Studies to Find Appropriate Spatial Focus}

A major concern in ecological studies should be the possibility that the relationships between the study organism and any habitat, environmental, or other species are not accurately measured because of the analytical focus at which they are measured $[12,20,39]$. To identify the analytical focus at which the abundance (or other measure) of a species responds to an aspect of the environment, the peak in the response curve (e.g., analytical focus with greatest correlation, $\mathrm{R}^{2}$, AIC) should be bracketed by foci encompassing larger and smaller areas (Fig. 3). The analytical focus at this peak is where this signal will be least attenuated. The signal itself is the strength of the relationship, the type of model that best fits (e.g., linear, polynomial, exponential), the retained predictor variables, and the coefficients and their signs. One possible guideline is to use foci at an order of magnitude above and below the suspected 'scale of response' [40••]. Using too small a range of foci could result in the researcher missing the response 'signal' [40••], or suggesting incorrect habitat relationships for their study species [39]. The strength of the relationship
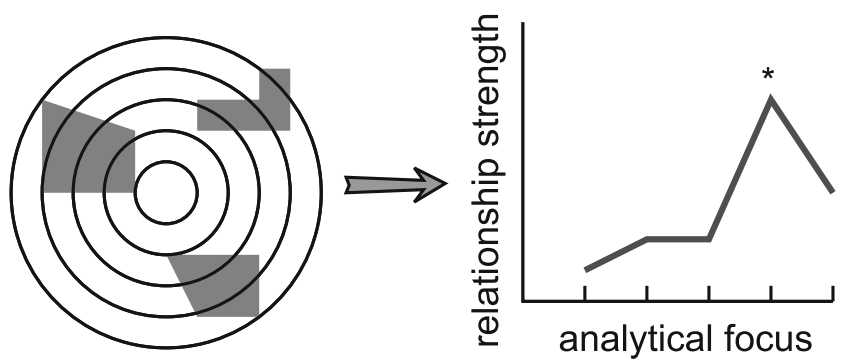

Fig. 3 Ecological studies using different analytical foci attempt to "tune in" to the ecological signal of organism-environment relationships. The analytical foci with the strongest relationship (e.g., greatest correlation, $\mathrm{R}^{2}, \mathrm{AIC}$ ) is where this signal will be least attenuated (asterisk). The signal itself is the nature of the relationship between the organism and the landscape between dependent and independent variables should peak and decline at analytical foci greater and less than the peak. The lack of a maximum response strength at an intermediate analytical focus, e.g., a monotonic increase, suggests that the appropriate analytical focus exists outside the range of values used [ $40 \cdot \bullet$. Regardless of the range of foci used, a lack of a detected relationship should not be assumed to suggest scaleinvariance [41].

Environmental independent variables that are variable only when using a very large analytical focus, such as climate, may not be able to be incorporated into a multi-scale type study because spatial replicates do not exist in sufficient number. The influence upon bark beetles of stand-, landscape-, and regional-level variables was recently studied using a multiscale spatial approach and a temporal analysis to examine effects of climate [42•]. Landscape connectedness of beetle populations and of host trees were both found to contribute to outbreaks. The use of a 23 year temporal extent allowed the researchers to examine variation in climate and detect its effect. This analysis would not have been possible using a single time interval because the distance over which the climate varied was greater than the spatial extent of the study. There may be other solutions yet to be discovered for limitations to multiscale studies, especially when variables of different dimensionality are used together.

Several spatial statistics and analytical frameworks have been adopted to relate species data to environmental variables to help identify the ecological neighborhood or at least the relevant analytical focus of from a range of foci (see $[11,16]$ for review). Traditional statistical methods such as generalized linear models and Spearman's correlation coefficient have been applied to the identification of the analytical focus. For example, Holland et al. [20] found the optimal analytical foci for longhorn beetle species by repeated linear regressions on subsets of survey data across a range of analytical foci. The relevant analytical focus was taken to be the radius with the greatest mean $r$ value.

Besides generalized linear models [20,43-45], several other analytical approaches have been used recently for 'multiscale' analysis: principal coordinates of neighbor matrices [46], multi-scale pattern analysis [47], Spearman's rank correlation [48-51], multidimensional scaling [52], and classification tree analysis [53]. Analytical approaches from other disciplines have also been borrowed for the identification of relevant analytical foci, such as geostatistical variograms [54]. As well, network modularity analysis has been used to identify the module, or set of tightly connected habitat patches or local populations, for examining ecological genetics [55]. The identified connected patches were then used to identify the 'critical scale' for animal movement and gene flow. Wagner and Fortin [56 $]$ proposed a conceptual framework for landscape genetics at four levels of analysis that are analogous to our analytical foci: node, link, neighborhood and boundary. 
Thus, the tool kit for identifying relevant analytical foci has greatly expanded to allow many different research questions to be asked and applicable types of data to be used.

The plant community at a location is influenced by many different environmental factors all acting at different 'scales' but Carlile et al. [57] suggest that this leads to an 'inherent scale' of the resulting community. They examined the progression of autocorrelation toward zero as the lag distance increases as an indicator of this inherent scale. A method for the detection of ecological neighborhood with similar theoretical underpinnings has been suggested [58]. The latter study used the same term 'inherent scale' to indicate what we would consider to be the ecological neighborhood. The researchers used simulations of a virtual species to show that residual spatial autocorrelation increased as the analytical focus was shifted further from the sought-after ecological neighborhood [58].

Several issues can arise in the identification of the analytical focus that best reflects the ecological neighborhood and strength of the species-environment relationship. The identification of the best analytical focus can be biased by pseudoreplication $[20,59 \bullet \bullet]$ spatial dependence $[58,59 \bullet$, $60]$ and the modifiable areal unit problem [61, 62•]. These issues and potential solutions are well-covered in the cited publications.

Species vary in the size of their ecological neighborhoods [e.g., 20]. Therefore, studies that use species richness or similar aggregate measures as a response variable will likely suffer attenuation in the strength of the measured response because the analytical focus chosen will be suboptimal for many of the species. Minimizing this signal loss will involve first identifying the relevant biological traits that determine the ecological neighborhood of the species involved. Species that respond to similarly-sized ecological neighborhoods may then be examined together at the appropriate common analytical focus. For example, species richness of beetles associated with dead aspen wood are more strongly related to the amount of dead deciduous wood within $3 \mathrm{~km}$ than to the amount within 1 or $2 \mathrm{~km}$ [43]. When the dependent variable of species richness is expanded to include saproxylic beetles not associated with aspen, the optimal spatial focus shifts away from $3 \mathrm{~km}$ with a concomitant decrease in the strength of the richness-landscape relationship. The shifts could be because aspen is a relatively minor component of the forest in the area and that dead aspen is an ephemeral resource, hence species using dead aspen are adapted to long distance dispersal compared to the other beetle species which use wood of tree species that are more widespread [43].

\section{Determinants of the Optimal Spatial Focus}

Movement distance is a major determinant of the ecological neighborhood for mobile organisms [7]. However, this neighborhood is not necessarily the ideal analytical focus to use because the ecological neighborhood is not the same as the ideal analytical focus. Jackson and Fahrig [10] used a simulation to find that analytical foci that measured landscape predictors at 4-9 times the mean lifetime dispersal distance led to better predictors of abundance. Their result highlights the importance of not selecting an analytical focus equal to the expected dispersal of the study organism. Conversely, the analytical focus that results in the best fit of organism data to landscape data should not be uncritically assumed to represent the dispersal or foraging distance of a species. Distances traveled for specific activities may translate into predictable analytical foci, but this remains to be studied. In particular, there will likely be several biological and ecological variables necessary besides movement distance necessary to explain a large proportion of the variance in the relationship between ecological neighborhood and analytical focus. The simulation study of Jackson and Fahrig [10] is a step in this direction.

Presence in a habitat patch may be determined by the vagility of uncommon dispersers whose movement is in the distal tail of the dispersal kernel [63]. Species' occurrence may therefore not be well modeled by population means. Occurrence may also be the result of many generations of dispersers, with habitats required at each generational step. Therefore, the landscape variation that corresponds to differences in occurrence may need to be measured at some multiple of mean dispersal distance [10]. The analytical focus found to reflect the relationship between occurrence and landscape connectivity may therefore be a multiple of the ecological neighborhood as determined by dispersal. Despite the difference between ecological neighborhood and analytical focus used to infer it, some studies with the spatial focus set at an estimate of mean dispersal have successfully found relationships between landscape parameters and study organism abundance (e.g., [64]). Such successes may occur because the magnitude of the landscape features measured at estimate of mean dispersal are representative of the composition and configuration of features in the larger surrounding areas as well. For example, if the abundance of leopard frogs in focal ponds is well predicted by the number of habitat patches within a distance corresponding to expected annual dispersal, this may indicate that the density and configuration of ponds within the replicated landscapes would remain similar if a larger spatial focus was used to measure the larger surrounding areas. The relationship may be weaker at this spatial focus in less homogeneous regions. Alternatively, the strength of the organism-environment relationship may not change significantly with a change in the focal scale of the study. The strength of relationship not varying with change in focal scale could occur either because the relationship is not biologically important as indicated by a low value of association, or because the relationship is important across a wide range of spatial foci. The latter hypothesis could be addressed by 
investigating larger and smaller foci. Local habitat conditions determine whether a species can use a patch or part of a landscape. The larger region surrounding the potential habitat will determine whether potential colonizers can reach the habitat. Therefore, suitable conditions and landscape connectivity (sensu [65]), are necessary, but in themselves insufficient, conditions for occurrence of a species. Habitat, necessary resources, and accessibility may be viewed as sequential filters to occurrence [66]. Habitat quality and habitat connectivity influenced abundance of several species of longhorned beetles at differently-sized ecological neighborhoods, as estimated with different spatial foci of best model fit among species [67]. The different species of longhorned beetles likely faced different ecological challenges, with each having a different ecological neighborhood. Furthermore, the surrounding area will determine the pool of potential colonizers [68] through the playing out of these same dynamics for other adjacent or overlapping landscapes [69]. Therefore, studies that incorporate both the local habitat conditions and the wider landscape should lead to greater predictability of occurrence or abundance [66]. For example, when Pearson [68] incorporated atsite vegetation and surrounding landscape vegetation classes, the resulting regression analyses explained $>80 \%$ of the variation for several species of over-wintering birds. Including only one of the independent environmental variables, or including local and landscape variables but measuring them at a single analytical focus, would have inappropriately weakened the strength of the measured relationship.

Organisms have ecological neighborhoods that vary in size depending on the activity or ecological interaction under consideration [7]. The size of the ecological neighborhood may vary from a small neighborhood influencing foraging decisions to larger neighborhoods encompassing large regions that determine nest location or natal dispersal success. However, determinants of relevant magnitudes of areas such as movement distances will also vary somewhat with landscape and among individuals. If there is a continuum in the type of activities undertaken, or at least in the relevant ecological neighborhood, then characterizing a species' movement or ecological neighborhood may require an infinite number of dispersal kernels as they are traditionally thought of. This will be especially important for animals that are not central place foragers (e.g., non-social insects). For animals that do not make repeated trips from a central location it will be more difficult to assign any segment of their lifetime movement into a discrete category. Therefore, it will be difficult to employ traditional dispersal kernels that have different moments for different activities. The probability of a given distance being covered by these organisms could be seen as a three dimensional surface, or even a probability density function of higher dimensionality, that represents a lifetime dispersal kernel that integrates probability over the range of activities performed or the time since birth (Fig. 4). The benefit of this more complex

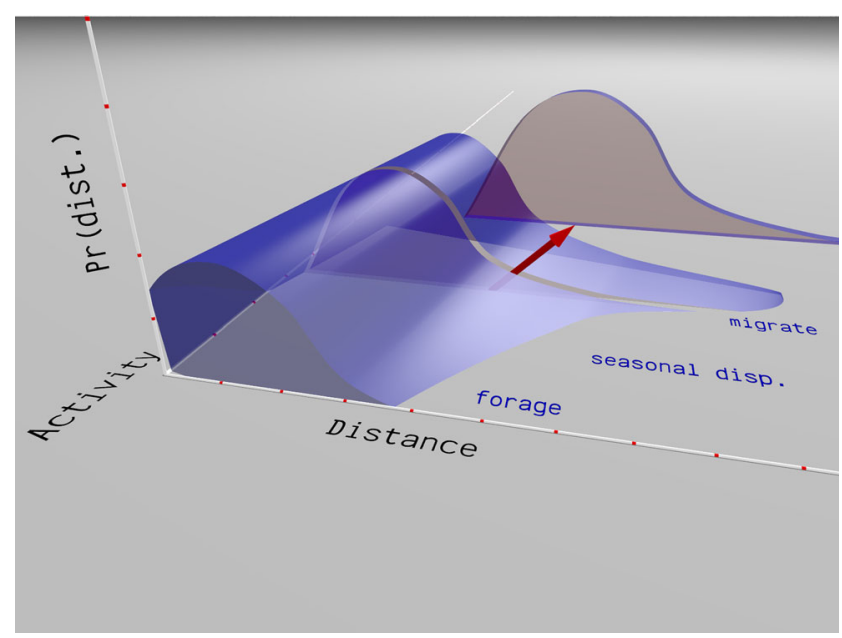

Fig. 4 A lifetime activity movement kernel for a species. The arrow indicates the more traditional view of a dispersal kernel is represented by a two dimensional slice from this distribution of movement distance

view will depend on the variance of the dispersal kernels for the different activities and interactions.

Eagles select areas to use for different activities according to variation at different response grains [15]. Foraging sites are selected based upon fine-grained variation in water depth, while selection of nesting sites is based upon canopy height variability within a small focus on the order of tens of meters. The difference between the observed response grains highlights the importance of using an analytical focus appropriate for the activity under study. Using a focus radius based upon home range $\left(5-35 \mathrm{~km}^{2} ;[15]\right)$ or lifetime dispersal may be appropriate for determining accessibility of potential habitat sites but would not be appropriate for uncovering local decisions regarding foraging and nesting.

The different ways that the landscape filters species occur at different spatial grains (sensu [7]) and should be examined at different analytical foci. However, an analytical focus should not be selected in isolation. The decisions or interactions that determine usage of an area are made at a given response grain and within a given ecological neighborhood, but they are constrained by the interactions at higher scales. Ecological studies will therefore be much more revealing if the different processes are considered together as a hierarchical system [70]. For example, habitat will remain unoccupied regardless of resources and environmental conditions if habitat fragmentation [71] or population extinction thresholds [72] prevent colonization. In addition, activities relevant at different response grains and in different ecological neighborhoods may interact and these interactions may even shift species' geographical ranges over time [73]. An understanding of the ecological context of the study species will therefore continue to be invaluable in interpreting results of multi-scale studies.

We have mainly discussed bottom-up influences of the landscape on the abundance of a species at a location or in a patch. However, top-down influences such as predation can 
also have a large influence on species' occurrence [73]. If this has a large effect on the variation in a prey species' abundance among locations, it will be important to consider. The influence of top-down effects on species' occurrence may be challenging to uncover because the ecological neighborhood of the relationship may have little to do with the movement of the prey species.

\section{The Future of Spatial Focus Studies}

An area ripe for investigation is the incorporation of some estimate of the number of dispersers, or population size as a proxy, in the determination of a patch-specific analytical focus around habitats. This variable-focus approach should allow for further 'tuning in on the ecological signal' because the area of interaction or distance to the places from which individuals originate is a function of the product of the number of dispersers and dispersal kernel. The number of organisms relevant to an ecological neighborhood can be seen as a number of random draws from the dispersal kernel (Fig. 4). If the ecological neighborhood is determined by some level of cumulative probability of dispersers reaching that distance, for example, then this will change with the number of dispersers as well as with the dispersal kernel. In metapopulation studies, the sizes of nearby patches are included in the determination of probability of exchanging individuals because size is a determinant of population size and persistence [74]. Using a variable analytical focus that depends upon some estimate of the number of dispersers may be seen as an extension of the mathematics of metapopulation studies into multiple-foci focal patch studies.

Another necessary advance in multi-scale studies is the development of methods to differentiate the signals returned through analyses of real ecological relationships and those created by the modifiable areal unit problem. Multi-scale studies as discussed here have greatly improved our understanding of relationships between organisms and their environments or the wider landscape. However, it remains possible that observed changes in relationships across spatial foci are an artifact of the grain at which things in nature are quantified [75].

In order to strengthen studies on the ecological neighborhood or analytical focus at which landscape-organism interactions occur, we must expand the use of experimental approaches in parallel with the predominant phenomenological approach. In this way, we can begin to formulate 'strong hypotheses' (sensu [76]) about the ecological relationships that determine the different aspects of the scale (e.g., [77]) of ecological interactions. Experiments on the mechanisms of ecological interactions will occur in concert with modeling studies, which are far ahead of experimental studies (e.g., [10]). Multiple foci studies similar to Wiens and Milne's [78] study of tenebrionid beetle movement in different plots may be a tractable way to begin examining the relationship between ecological neighborhood and analytical focus. We must remain focused on the biology underlying these relationships [15]. Multi-scale studies may have a proximate goal of determining the factors altering the appropriate analytical focus. However, their ultimate goal is to achieve a greater understanding of the ecological interactions under study through the use of appropriately sized study replicates. Greater understanding of the ecological interactions will thus strengthen the hypothesis under study by eliminating one source of type II error.

Finally, we now have the tools to extend the use of multiscale focal patch studies further into landscape management to increase ecological sustainability. For example, a multi-scale approach was recently used to examine the 'scale of effect' of restorations in forested landscapes [79]. A multiple analytical foci study examined the damage done by monk parakeets to crops within fields and how this damage level was related to characteristics of the field and the surrounding landscape within three analytical foci $(1000,3000$ and $5000 \mathrm{~m})$ [80]. This study found different sets of variables explained the damage at the different foci. These results suggest field cultural practices that may reduce crop damage, and also landscape features within particular analytical foci that will influence crop damage as well.

Altering the balance between pest and beneficial species using relatively benign methods such as conservation biological control are to be preferred over less sustainable management options. While it will only be logistically possible to alter entire landscapes to this end in a few select cases, ecologists can recommend particular practices that are best suited to given landscape contexts. One factor in such recommended practices could be how given landscapes are predicted to support pest species or exotic invasive species versus species that are benign or providers of ecosystem services. For example, studies have shown that devastating bark beetles such as the southern pine beetle Dendroctonus frontalis have a different dispersal kernel, at least at the tail of the distribution, than their checkered beetle predator Thanasimus dubius [81]. There will be many other parameters of dispersal behavior, habitat needs, foraging behavior, and landscape thresholds and connectivity that are integrated into the optimal spatial foci for such species. If landscape parameters that determine occurrence of such pest and beneficial species can be found by using multiple spatial foci landscape studies, these parameters could help guide appropriate management for tipping the balance between the tree-killing pest beetle and its beneficial (to human interests) predator. For example, Ryall and Fahrig [82] found that landscapes with less pine forest had focal patches with greater abundances of the bark beetle Ips pini and lower abundances of their predators. Determining the spatial foci that individually reveal ecological interactions for bark beetle and predator abundance could lead to recommendations of 'good landscapes' and 'bad landscapes' 
for future pine forests. Land use recommendations based on the likelihood of supporting pest and beneficial species will be complicated by the effects of climate differentially affecting the pests and their predators. Climate not only varies across very large areas, but also has a much greater impact on pest wood-borers by causing tree stress than on the predators. For example, recent work on an exotic invasive wood-borer suggests that natural enemies usually keep wood-borers in check, but that reductions in tree health can allow the wood-borer populations to outstrip the ability of natural enemies to control them [83].

There remain several determinants of relevant analytical foci to be investigated. Throughout future multiple scale research, it will be useful to keep in mind that the ecological neighborhood and our refinements to it are constructs that aid us by focusing our investigations at appropriate scales. Organisms are not 'influenced by spatial scale' any more than plants are influenced by plant density per se. To be sure, plants are influenced by neighboring plants and lack of resources. Similarly, mobile species are influenced by many ecological interactions that occur across different distances. Analytical foci are simply lenses that may be varied in size to allow the results of ecological studies to best represent the studied species-environment relationship (Fig. 2). They are a step toward discovering the important mechanisms driving abundances rather than phenomena that themselves impact abundance. By using consistent and clear terminology and remaining focused on the ecological mechanisms at play we can keep 'scale' in its rightful place in the ecologist's toolbox rather than lurking in the fields and forests amongst our study species.

Acknowledgments We thank Ian Kaplan, Cliff Sadof, and two anonymous reviewers for helping us improve upon earlier versions of this paper.

\section{Compliance with Ethical Standards}

Conflict of Interest On behalf of all authors, the corresponding author states that there is no conflict of interest.

Human and Animal Rights and Informed Consent This article contains no studies with human or animal subjects performed by the authors.

\section{References}

Papers of particular interest, published recently, have been highlighted as:

- Of importance

- Of major importance

1. Schneider DC. The rise of the concept of scale in ecology. Bioscience. 2001;51:545-53.
2. Chave J. The problem of pattern and scale in ecology: what have we learned in 20 years? Ecol Lett. 2013;16:4-16.

3. Wiens JA. Pattern and process in grassland bird communities. Ecol Monogr. 1973;43:237-70.

4. Wiens JA. Spatial scaling in ecology. Funct Ecol. 1989;3:385-97.

5. Wiens JA, Addicott JF, Case TJ, Diamond J. Overview: the importance of spatial and temporal scale in ecological investigations. Community Ecol. 1986;1:145-53.

6. Wiens JA, Rotenberry JT, Van Horne B. Habitat occupancy patterns of North American shrubsteppe birds: the effects of spatial scale. Oikos. 1987;48:132-47.

7. Addicott JF, Aho JM, Antolin MF, Padilla DK, Richardson JS, Soluk DA. Ecological neighborhoods: scaling environmental patterns. Oikos. 1987:49:340-6.

8. Levin SA. The problem of pattern and scale in ecology: the Robert H. MacArthur award lecture. Ecol Soc Am. 1992;73:1943-67.

9. Sutherland WJ, Freckleton RP, Godfray HCJ, Beissinger SR, Benton T, Cameron DD, et al. Identification of 100 fundamental ecological questions. J Ecol. 2013;101:58-67.

10.• Jackson HB, Fahrig L. What size is a biologically relevant landscape? Landsc Ecol. 2012;27:929-41.

11. Dray S, Pélissier R, Couteron P, Fortin M-J, Legendre P, Peres-Neto $\mathrm{P}$, et al. Community ecology in the age of multivariate multiscale spatial analysis. Ecol Monogr. 2012;82:257-75.

12. Brennan JM, Bender DJ, Contreras TA, Fahrig L. Focal patch landscape studies for wildlife management: optimizing sampling effort across scales. In: Liu J, Taylor WW, editors. Integrating landscape ecology into natural resource management. 1st ed. Cambridge: Cambridge University Press; 2002. p. 68-91.

13. Schneider DC. Quantitative ecology: spatial and temporal scaling. London: Academic Press; 1994.

14. Turner MG, Dale VH, Gardner RH. Predicting across scales: theory development and testing. Landsc Ecol. 1989;3:245-52.

15. Thompson CM, McGarigal K. The influence of research scale on bald eagle habitat selection along the lower Hudson River, New York (USA). Landsc Ecol. 2002;17:569-86.

16. Dungan JL, Perry JN, Dale MRT, Legendre P, Citron-Pousty S, Fortin M, et al. A balanced view of scale in spatial statistical analysis. Ecography. 2002;25:626-40.

17. Anderson TM, Metzger KL, McNaughton SJ. Multi-scale analysis of plant species richness in Serengeti grasslands. J Biogeogr. 2007:34:313-23.

18. Scheiner SM, Cox SB, Willig M, Mittelbach GG, Osenberg C, Kaspari M. Species richness, species-area curves and Simpson's paradox. Evol Ecol Res. 2000;2:791-802.

19. Lechner AM, Langford WT, Bekessy SA, Jones SD. Are landscape ecologists addressing uncertainty in their remote sensing data? Landsc Ecol. 2012;27:1249-61.

20. Holland JD, Bert DG, Fahrig L. Determining the spatial scale of species' response to habitat. Bioscience. 2004;54:227-33.

21. de Knegt HJ, van Langevelde F, Skidmore AK, Delsink A, Slotow R, Henley S, et al. The spatial scaling of habitat selection by African elephants. J Anim Ecol. 2011;80:270-81.

22. Comfort EJ, Clark DA, Anthony RG, Bailey J, Betts MG. Quantifying edges as gradients at multiple scales improves habitat selection models for northern spotted owl. Landsc Ecol. 2016. doi:10.1007/s10980-015-0330-1.

23. Morris DW. Ecological scale and habitat use. Ecology. 1987;68: 362-9.

24. Molofsky J, Bever JD, Antonovics J, Newman TJ. Negative frequency dependence and the importance of spatial scale. Ecology. 2002;83:21-7.

25. Johnson RK, Goedkoop W, Sandin L. Spatial scale and ecological relationships between the macroinvertebrate communities of stony habitats of streams and lakes. Freshw Biol. 2004;49:1179-94. 
26. Morris DW. Scales and costs of habitat selection in heterogeneous landscapes. Evol Ecol. 1992;6:412-32.

27. Wright S. Isolation by distance. Genetics. 1943;28:114-38.

28. Wright $\mathrm{S}$. Isolation by distance under diverse systems of mating. Genetics. 1946;31:39-59.

29. Brown JH, Kodric-Brown A. Turnover rates in insular biogeography: effect of immigration on extinction. Ecology. 1977;58:445-9.

30. Southwood TRE. Habitat, the templet for ecological strategies? J Anim Ecol. 1977;46:337-65.

31. Price PW. Evolutionary biology of parasites. Princeton: Princeton University Press; 1980.

32. Antonovics J, Levin DA. The ecological and genetic consequences of density-dependent regulation in plants. Annu Rev Ecol Syst. 1980;11:411-52.

33. Mack RN, Harper JL. Interference in dune annuals: spatial pattern and neighbourhood effects. J Ecol. 1977;65:345-63.

34. Schneider DC, Piatt JF. Scale-dependent correlation of seabirds with schooling fish in a coastal ecosystem. Mar Ecol Prog Ser. 1986;32:237-46.

35. Mazerolle MJ, Villard M-A. Patch characteristics and landscape context as predictors of species presence and abundance: a review. Ecoscience. 1999;6:117-24.

36. Thornton DH, Branch LC, Sunquist ME. The influence of landscape, patch, and within-patch factors on species presence and abundance: a review of focal patch studies. Landsc Ecol. 2011;26: $7-18$.

37. Lynch JF, Whigham DF. Effects of forest fragmentation on breeding bird communities in Maryland. USA Biol Conserv. 1984;28:287324.

38. Fortin MJ. James PM a, MacKenzie A, Melles SJ, Rayfield B. Spatial statistics, spatial regression, and graph theory in ecology. Spat Stat. 2012;1:100-9.

39. Wasserman TN, Cushman SA, Wallin DO, Hayden J. Multi Scale habitat relationships of Martes americana in northern Idaho, U.S.A. Environmental Sciences Faculty Publications. Paper 20. 2012.

40.• Miguet P, Jackson HB, Jackson ND, Martin AE, Fahrig L. What determines the spatial extent of landscape effects on species? Landsc Ecol. 2015. doi:10.1007/s10980-015-0314-1. A comprehensive review of hypothesized drivers of scales of response.

41. Gunton RM, Pöyry J. Scale-specific spatial density-dependence in parasitoids: a multi-factor meta-analysis. Funct Ecol. 2015. doi:10.1111/1365-2435.12627.

42. Seidl R, Müller J, Hothorn T, Bässler C, Heurich M, Kautz M. Small beetle, large-scale drivers: how regional and landscape factors affect outbreaks of the European spruce bark beetle. J Appl Ecol. 2015. doi:10.1111/1365-2664.12540. Example of a multiscale study that demonstrates the importance of conducting studies at multiple spatial and temporal scales.

43. Jacobsen RM, Sverdrup-Thygeson A, Birkemoe T. Scale-specific responses of saproxylic beetles: combining dead wood surveys with data from satellite imagery. J Insect Conserv. 2015;19:1053-62.

44. Seavy NE, Viers JH, Wood JK. Riparian bird response to vegetation structure: a multiscale analysis using LiDAR measurements of canopy height. Ecol Appl. 2009;19:1848-57.

45. Fisher JT, Anholt B, Volpe JP. Body mass explains characteristic scales of habitat selection in terrestrial mammals. Ecol Evol. 2011;1:517-28.

46. Borcard D, Legendre P. All-scale spatial analysis of ecological data by means of principal coordinates of neighbour matrices. Ecol Model. 2002;153:51-68.

47. Jombart T, Dray S, Dufour AB. Finding essential scales of spatial variation in ecological data: a multivariate approach. Ecography. 2009;32:161-8.
48. Cardoso P, Aranda SC, Lobo JM, Dinis F, Gaspar C, Borges PAV. A spatial scale assessment of habitat effects on arthropod communities of an oceanic island. Acta Oecol. 2009;35:590-7.

49. Duren KR, Buler JJ, Jones W, Williams CK. An improved multiscale approach to modeling habitat occupancy of Northern bobwhite. J Wildl Manag. 2011;75:1700-9.

50. Irvin E, Duren KR, Buler JJ, Jones W, Gonzon AT, Williams CK. A multi-scale occupancy model for the grasshopper sparrow in the Mid-Atlantic. J Wildl Manag. 2013;77:1564-71.

51. Guerena KB, Castelli PM, Nichols TC, Williams CK. Spatiallyexplicit land use effects on nesting of Atlantic Flyway resident Canada geese in New Jersey. Wildl Biol. 2014;20:115-21.

52. Andrade-Núñez MJ, Aide TM. Effects of habitat and landscape characteristics on medium and large mammal species richness and composition in northern Uruguay. Zool. 2010;27:909-17.

53. Albanese G, Davis CA, Compton BW. Spatiotemporal scaling of North American continental interior wetlands: implications for shorebird conservation. Landsc Ecol. 2012;27:1465-79.

54. Schaefer JA, Mayor SJ. Geostatistics reveal the scale of habitat selection. Ecol Model. 2007;209:401-6.

55. Fletcher Jr. RJ, Revell A, Reichert BE, Kitchens WM, Dixon JD, Austin JD. Network modularity reveals critical scales for connectivity in ecology and evolution. Nat Commun. 2013; 4: doi:10.1038 /ncomms3572.

56. Wagner HH, Fortin MJ. A conceptual framework for the spatial analysis of landscape genetic data. Conserv Genet. 2013;14:25361. A conceptual framework for landscape genetic data with explanation of analogous study units in traditional landscape ecology and landsape genetics.

57. Carlile DW, Skalski JR, Batker JE, Thomas JM, Cullinan VI. Determination of ecological scale. Landsc Ecol. 1989;2:203-13.

58. de Knegt HJ, van Langevelde F, Coughenour MB, Skidmore AK, de Boer WF, Heitkönig IMA, et al. Spatial autocorrelation and the scaling of species-environment relationships. Ecology. 2010;91: 2455-65.

59.• Jackson HB, Fahrig L. Are ecologists conducting research at the optimal scale? Glob Ecol Biogeogr. 2014;24:52-63. An extensive review of multi-scale biological studies. Suggestions made on identifying optimal scale, and observation that response scale may be outside the range of scales being studied in several studies.

60. Legendre P, Fortin M. Spatial pattern and ecological analysis. Vegetatio. 1989;80:107-38.

61. Jargowsky PA. Ecological Fallacy. Encycl Soc Meas. 2005;715-22.

62. Sandel B. Towards a taxonomy of spatial scale-dependence. Ecography. 2014;38:358-69. Clarification of terms used in multi-scale studies and studies of spatial scale-dependence. Results of not using appropriate range of some aspect of spatial scale explained.

63. Liebhold AM, Tobin PC. Population ecology of insect invasions and their management. Annu Rev Entomol. 2008;53:387-408.

64. Pope SE, Fahrig L, Merriam HG. Landscape complementation and metapopulation effects on leopard frog populations. Ecology. 2000;81:2498-508.

65. Taylor PD, Fahrig L, Henein K, Merriam G. Connectivity is a vital element of landscape structure. Oikos. 1993;68:571-3.

66. Jackson HB. Baum K a., Cronin JT. From logs to landscapes: determining the scale of ecological processes affecting the incidence of a saproxylic beetle. Ecol Entomol. 2012;37:233-43.

67. Yang S. Landscape scaling and occupancy modeling with Indiana longhorned beetles (Coleoptera: Cerambycidae) [PhD Thesis]. West Lafayette: Purdue University; 2010.

68. Pearson SM. The spatial extent and relative influence of landscapelevel factors on wintering bird populations. Landsc Ecol. 1993;8:318. 
69. Karger DN, Cord AF, Kessler M, Kreft H, Kühn I, Pompe S, et al. Delineating probabilistic species pools in ecology and biogeography. Glob Ecol Biogeogr. 2016. doi:10.1111/geb.12422.

70. Benton TG, Bowler DE. Linking dispersal to spatial dynamics. In: Clobert J, Baguette M, Benton TG, editors. Dispersal Ecology and Evolution. 1st ed. Oxford: Oxford University Press; 2012. p. 25165 .

71. Holland JD. Glycobius speciosus (Say)(Coleoptera: Cerambycidae) has been extirpated from much of Midwestern USA. Coleopt Bull. 2009;63:54-61.

72. Holland JD, Fahrig L, Cappuccino N. Fecundity determines the extinction threshold in a Canadian assemblage of longhorned beetles (Coleoptera: Cerambycidae). J Insect Conserv. 2005;9:109-19.

73. Yackulic CB, Ginsberg JR. The scaling of geographic ranges: implications for species distribution models. Landsc Ecol. 2016. doi:10.1007/s10980-015-0333-y.

74. Hanski I. A practical model of metapopulation dynamics. J Anim Ecol. 1994;63:151-62.

75. Lechner AM, Langford WT, Jones SD, Bekessy SA, Gordon A. Investigating species-environment relationships at multiple scales: differentiating between intrinsic scale and the modifiable areal unit problem. Ecol Complex. 2012;11:91-102.
76. Platt JR. Strong inference. Science. 1964;146:347-53.

77. Holland JD, Fahrig L, Cappuccino N. Body size affects the spatial scale of habitat-beetle interactions. Oikos. 2005;110:101-8.

78. Wiens JA, Milne BT. Scaling of "landscapes" in landscape ecology, or, landscape ecology from a beetle's perspective. Landsc Ecol. 1989;3:87-96.

79. Crouzeilles R, Curran M. Which landscape size best predicts the influence of forest cover on restoration success?-A global metaanalysis on the scale of effect. J Appl Ecol. 2016. doi:10.1111 /1365-2664.12590.

80. Canavelli SB, Branch LC, Cavallero P, González C, Zaccagnini ME. Multi-level analysis of bird abundance and damage to crop fields. Agric Ecosyst Environ. 2014;197:128-36.

81. Cronin JT, Reeve JD, Wilkens R, Turchin P. The pattern and range of movement of a checkered beetle predator relative to its bark beetle prey. Oikos. 2000;90:127-38.

82. Ryall KL, Fahrig L. Habitat loss decreases predator-prey ratios in a pine-bark beetle system. Oikos. 2005;110:265-70.

83. MacQuarrie CJK, Scharbach R. Influence of mortality factors and host resistance on the population dynamics of emerald ash borer (Coleoptera: Buprestidae) in urban forests. Environ Entomol. 2015;44:160-73. 